\title{
Abnormal Expression of NOS3 and Association with Clinical Outcome are Highly Cancer Dependent as Revealed through Pan-Cancer Analysis
}

\section{Dan Zou}

China Medical University First Hospital

Fei Lv

China Medical University First Hospital

Yi Yang

China Medical University

Chunjiao Yang

China Medical University First Hospital

\section{Yang Chen}

China Medical University First Hospital

Zi Jin

Shenyang Fifth People's Hospital

Jinpeng Zhou

China Medical University First Hospital

Yang Jiang

China Medical University First Hospital

Zhitao Jing

China Medical University First Hospital

Ye Zhang ( $\sim$ yzhang21@cmu.edu.cn )

Department of Medical Oncology https://orcid.org/0000-0002-7238-6964

Research article

Keywords: NOS3, pan-cancer, gene expression

Posted Date: December 16th, 2019

DOI: https://doi.org/10.21203/rs.2.19006/v1

License: (1) (1) This work is licensed under a Creative Commons Attribution 4.0 International License.

Read Full License 


\section{Abstract}

Background: NOS3 (endothelial NOS, eNOS) is a member of the nitric oxide synthase (NOS) enzyme family, mainly participating in nitric oxide (NO) generation. NOS3 has been reported to inhibit apoptosis and promote angiogenesis, proliferation, and invasiveness. However, the expression pattern of NOS3 and its diagnostic and prognostic potential has not been investigated in a pan-cancer perspective.

Methods: In this research, data from the Genotype-Tissue Expression (GTEx), the Cancer Genome Atlas (TCGA), the Cancer Cell Line Encyclopedia (CCLE) and the Cancer Therapeutics Response Portal (CTRP) were employed and NOS3 expression was comprehensively analysed in normal tissues, cancer tissues and cell lines. Its relationship with clinical phenotypes and drug responses was also analysed.

Results: In normal tissues, NOS3 was expressed at the highest levels in the spleen and the lowest levels in the blood. Compared with the corresponding normal tissues, its expression in tumour was significantly increased in seven tumour types but decreased in eight tumour types. And NOS3 expression was positively or negatively related to tumour stage and overall survival of patients depending on the tumour types. The expression of NOS3 was related to the response to 'SR8278'.

Conclusions: NOS3 was differentially expressed in tumour tissues, and had prognosis value in some tumour types. And its correlation to drug response warrants further investigation.

\section{Background}

NOS3 (endothelial NOS, eNOS) is a member of the nitric oxide synthase (NOS) enzyme family, which is a cluster of catalytic enzymes that mainly participate in nitric oxide (NO) generation (1). The NOS3 protein is encoded by the NOS3 gene, located on chromosome 7q36.1. Usually, NOS3 protein is constitutively expressed in cells in an inactive state. Following the increase in calcium (Ca2+) concentration in cells, it can be activated by combining with the CaM protein. In addition, the direct combination of NOS3 with caveolin-1 (CAV-1) and heat shock protein 90 (HSP90) and the phosphorylation (Ser-1177) of NOS3 by $\mathrm{PI} 3 \mathrm{~K} /$ Akt signalling can modulate the activity of NOS3 protein (2-4).

NOS3 protein was initially found to participate in NO generation, mainly in endothelial cells, and is associated with cardiovascular diseases such as hypertension, atherosclerosis, and diabetes mellitus (5). In recent years, NOS3 has been found to play various roles in malignant tumours, such as inhibiting apoptosis and promoting angiogenesis, proliferation, invasiveness, and immunosuppression (6-8). Circulating NOS3 levels were significantly correlated with progression-free survival and overall survival (OS) of metastatic colorectal cancer patients (9). Another research in mesenchymal colorectal cancer patients reported that NOS3 upregulation occurs after Apc loss which was associated with poor prognosis (10). In breast cancer, the increasing expression of NOS3 was reported to be a pro-angiogenic factor (11). It was found to exert pro-angiogenesis function as a downstream molecule of the $\mathrm{PI3K} / \mathrm{Akt} / \mathrm{mTOR}$ pathway (12). NOS3-NO-sGC-cGMP signalling was reported to enhance the migration and invasion of breast cancer cells (13). In pancreatic cancer, NOS3 promoted tumour maintenance 
through the PI3K-Akt-NOS3-RAS (wild type) pathway (14). NOS3 inhibition by the inhibitor N(G)-nitro-Larginine methyl ester (L-NAME) could suppress pancreatic ductal adenocarcinoma cancer (PDAC) tumour growth (15). Yu et al. reported that NOS3 activation promoted increased the antiandrogen-resistant growth of prostate cancer (PCa) cells through NO-mediated AR suppression (16). Nanni et al. found that NOS3 might remodel the response of PCa cells to oestrogen (E2) and hypoxia stimuli through its combination with oestrogen receptor beta (ER $\beta$ ) and hypoxia inducible factors (HIFs), resulting in poor prognosis in PCa patients (17). In addition, another study reported that NOS3 downregulated miR-34a expression, inducing overexpression of SIRT1 in aggressive PCa, and in turn, SIRT1 stimulated the activation of NOS3. E2 induced ER signalling could promote this interaction (18). Trachootham et al. found that nontumourigenic epithelial cells from oral and ovarian tissue could be induced to become invasive in stroma containing p53-deficient fibroblasts, through NOS3/RNS/ICAM1 signalling (19). In addition, NOS3 was found to participate in oncogenic inflammation and immunosuppression of tumours through NOTCH1-PI3K/AKT-NOS3 axis (20). NOS3 expression inhibition was involved in cervical cancer cell sensitivity to radiotherapy (21).Additionally, many studies have reported that NOS3 gene polymorphisms are associated with risk for cancer progression, cancer susceptibility and drug response (22-24). However, several studies have reported that NOS3 might play an anticancer role in malignant tumours. Research by Smeda et al. reported that NOS3 activity and phosphorylation reduction was an early event in the lung metastasis of breast cancer, preceding the onset of the mesenchymal phenotype (EndMT) (25). NOS3 participated in the enhancement of Taxol chemosensitivity with astragaloside IV treatment in breast cancer as a downstream target of caveolin-1(26). These studies suggest that NOS3 may perform multiple functions depending on different tumour types, and genetic background. Studies on NOS3 expression in tumours are still scarce, and the functions of NOS3 in tumour pathogenesis are not comprehensively understood.

By applying data from the Genotype-Tissue Expression (GTEx; https://www.gtexportal.org/home/), The Cancer Genome Atlas (TCGA; https://portal.gdc.cancer.gov/) and the Cancer Cell Line Encyclopedia (CCLE; https://portals.broadinstitute.org/ccle/), the expression level of NOS3 in 30 different normal human tissues and 33 different tumours types, as well as the corresponding normal tissues and 1457 cancer cell lines was systematically analysed. We investigated the relationship between NOS3 expression and the clinical phenotypes of patients for all cancers and then separately for each cancer type.

Subsequently, NOS3 expression levels across different cell clines were also evaluated by using data from the CCLE. The correlation between NOS3 expression level in 664 cancer cells and cell response to 544 drugs was analysed to explore the potential of NOS3 as a therapeutic target. These analyses revealed that the expression level and clinical significance of NOS3 varied depending on the tissue or cancer type. NOS3 expression in several cancers was significantly different than that in corresponding normal tissues and showed implications for tumour stage and overall survival of patients. In addition, our results have inspired us regarding which tumours and therapeutic drugs to focus on for our studies.

\section{Methods}




\section{Download and analysis of TCGA and GTEx datasets}

TOIL GTEx and TCGA (primary tumour and normal tissues) gene expression RNA-seq data (IlluminaHiSeq: log2-normalized_count + 1) and TCGA phenotype data, containing 9359 TCGA tumour tissues, 727 TCGA normal tissues and 7792 GTEx normal tissues, were obtained from UCSC Xena (https://xena.ucsc.edu/). TOIL reprocesses raw GTEx and TCGA RNA-seq data to correct for batch effects and to allow for the merging of samples across GTEx and TCGA datasets for pan-analyses (27). To analyse the differential expression of NOS3 between TCGA tumours and normal tissues, t-test was applied for tumour types with at least two normal tissues. The median gene expression level was employed to calculate the fold change. Then, the log2-fold change (cancer versus normal) was employed as the $x$-axis and - $\log 10 p$-value was employed as the $y$-axis to produce a Volcano plot for each tumour type. NOS3 expression levels among different tumour stages (TNM stage) were assessed by t-test (for two groups) and ANOVA analyses (for three and more groups). To assess the relationship of NOS3 expression to overall survival (diagnosis to death), Cox proportional hazards models were employed. Patients who survived with no evidence of disease at the time of analysis were investigated at the date of last follow up. The median expression level in each tumour type was used as a threshold to divide patients into two groups. Kaplan-Meier survival analyses and the log-rank test were applied to compare survival differences in the two groups for each cancer type. A forest plot was constructed to visually display the hazard ratio (HR) and 95\% confidence interval for each tumour type. The 95\% confidence interval without containing 1, was considered a significant correlation. An HR value more than 1 indicated that increased NOS3 expression was related to poor prognosis (less than 1 is favourable), and the HR value represented an increased (or decreased) risk for every doubling expression of NOS3.

\section{Cell lines NOS3 expression and drug response}

NOS3 mRNA expression, promoter DNA methylation and copy number data were downloaded from the Cancer Cell Line Encyclopedia (CCLE, https://portals.broadinstitute.org/ccle/), which contained RNA-seq data, DNA methylation data from the matching reduced representation bisulfite sequencing (RRBS) and copy number data of 1457 human cancer cell lines. The drug response data were obtained from the Cancer Therapeutics Response Portal (CTRP, https://portals.broadinstitute.org/ctrp.v2.1/), which contained the responses of 664 cell lines to 482 drugs. NOS3 expression levels among different cell lines of different cancer types were investigated, and its relation to promoter DNA methylation and copy number was evaluated by Spearman correlation analysis. In addition, Spearman correlation analyses was also performed to evaluate the association of NOS3 expression with drug responses (area under the curve, AUC) first for all cell lines together and then separately for each cancer cell line type with at least 15 cell lines.

For all statistical analyses, a p-value less than 0.05 was considered to be statistically significant. All statistical analyses and visualization were accomplished by using GraphPad Prism 7 and R software (R version 3.6.0). 


\section{Results}

\section{NOS3 mRNA expression in various normal tissues and tumours.}

To comprehensively analyse NOS3 expression and distribution in human normal tissues and tumour tissues, we first analysed NOS3 mRNA expression level in 30 different normal tissues from GTEx and 33 different tumour tissues from Xena (https://xenabrowser.net/). The expression of NOS3 was highly variable across different normal tissues (Figure 1A) and tumour tissues (Figure 1B). In normal tissues, the median NOS3 expression levels varied from 5.624 (blood) to 12.8 (spleen). Tissues with the highest NOS3 expression were spleen (12.8 \pm 1.391$)$, heart (10.64 \pm 1.099$)$, testis $(10.59 \pm 0.532)$, adipose tissue (10.5 \pm 0.8709$)$, and breast (10.42 \pm 0.8376$)$. Tissues with the lowest NOS3 expression were blood (5.624 \pm 1.325$)$, skin (7.904 \pm 1.753$)$, pancreas (8.363 \pm 1.159$)$, muscle (8.415 \pm 1.022$)$, and ovary (8.453 \pm 0.9167$)$. In tumour tissues, NOS3 expression levels varied from 9.85 (stomach adenocarcinoma, STAD) to 5.071 (acute myeloid leukeamia, LAML). Tumour tissues with the highest NOS3 expression were STAD (9.85 \pm 1.018$)$, kidney renal clear cell carcinoma (KIRC, 9.848 \pm 0.9791$)$, pancreatic adenocarcinoma (PAAD, 9.297 \pm 0.8167$)$, testicular germ cell tumours (TGCT, 9.285 \pm 0.6801 ), and oesophageal carcinoma (ESCA, 9.275 \pm 1.208$)$. Tumour tissues with the lowest NOS3 expression were LAML (5.071 \pm 1.591$)$, kidney renal papillary cell carcinoma (KIRP, 7.173 \pm 1.14$)$, uveal melanoma (UVM, 7.278 \pm 0.9764 ), cervical and endocervical cancers (CESC, 7.388 \pm 1.054 ), and thymoma (THYM, $7.439 \pm 0.987)$.

\section{NOS3 is differentially expressed in various tumours and their corresponding normal tissues.}

We analysed NOS3 mRNA expression levels across tumours and their corresponding normal tissues in 21 tumour types that had three or more normal tissues data (Figure 2A). NOS3 was expressed at certain level in all tumours and normal tissues. NOS3 mRNA expression in 7 of 21 tumour types, colon adenocarcinoma (COAD), head and neck squamous cell carcinoma (HNSC), KIRC, prostate adenocarcinoma (PRAD), rectum adenocarcinoma (READ), STAD, and thyroid carcinoma (THCA), was much higher than that in corresponding normal tissues, with statistical significance. In 8 of 21 tumour types, bladder urothelial carcinoma (BLCA), breast invasive carcinoma (BRCA), CESC, kidney chromophobe (KICH), KIRP, liver hepatocellular carcinoma (LIHC), PAAD, and uterine corpus endometrial carcinoma (USEC), NOS3 mRNA expression was lower than that in corresponding normal tissues. The different expression levels among tumours indicated that NOS3 might play an anti-tumour or pro-tumour role depending on the tumour type. Further analyses of fold change between tumour and corresponding normal tissues found that NOS3 was most differentially expressed in CESC, with a more than 4-fold decrease in tumour tissues ( $\log 2 \mathrm{FC}=2.92, \mathrm{p}<0.0001$ ) (Figure 2B, 2C). NOS3 expression in KIRP tumour tissues was also 4-fold decreased compared with corresponding normal tissues (log2 $\mathrm{FC}=2.36$, $p<0.0001)$. In addition, our analysis found that NOS3 mRNA expression levels in CESC and KIRP were relatively low among the 33 tumour types (Figure 1B). However, it was worth noting that there were only three corresponding normal tissues for CESC, which in some content might result in a deviation in the differential expression analyses. In addition, NOS3 expression in both STAD and READ tumour tissues 
was more than 2-fold greater than that in the corresponding normal tissues. Surprisingly, STAD tumour tissues had the highest NOS3 expression level of the 33 tumour types.

\section{Association between NOS3 mRNA expression and clinical phenotypes.}

We analysed the association between NOS3 expression and tumour stage in 25 tumour types that had stage information in TCGA. First, we analysed NOS3 expression across four stages by means of ANOVA analyses on 25 tumours combined and found that NOS3 was differentially expressed across stages (Figure 3A). Patients in stage III expressed the highest NOS3 levels. Subsequently, stages I and II were combined as early stage, and stages III and IV were combined as advanced stage. T-test analyses showed that NOS3 expression was higher in the advanced stage (Figure 3B, $p<0.0001$ ). Further analyses of different tumour types found that BLCA, BRCA, COAD, ESCA, KIRP, and skin cutaneous melanoma (SKCM) tumours in advanced tumour stage expressed higher NOS3 mRNA levels. In contrast, KIRC and THCA tumours in the advanced tumour stage expressed lower NOS3 mRNA levels (Figure 3C).

To analyse the relationship between NOS3 expression and overall survival of tumour patients, log-rank test was performed in 33 tumour types (Figure 4A, B). We found that NOS3 mRNA expression level was related to a worse prognosis inpatients with KIRP [median survival (low versus high): not reached versus 2941, $p=0.0043$, hazard ratio $(H R)=2.441$, mesothelioma (MESO, median survival: 732 versus 456.5, $p=0.0032$, HR=1.945), and STAD (median survival: 1686 versus $801, p=0.0133385, \mathrm{HR}=1.394$ ). While it was related to a better prognosis in patients with KIRC [median survival: 2386 versus not reached, $p=0.0133, \mathrm{HR}=0.6835$ ] and lung adenocarcinoma (LUAD, median survival: 1454 versus $1622, p=0.0284$, $\mathrm{HR}=0.7228$ ). Moreover, KIRP had the largest HR with a 95\% confidence index between 1.35 and 4.414 . Besides, we found that high expression level of NOS3 was associated with both advanced tumour stage and worse prognosis in KIRP patients and was associated with early tumour stage and better prognosis in KIRC patients, indicating the potential clinical significance of NOS3 in KIRP and KIRC.

\section{NOS3 mRNA expression in tumour cell lines.}

Considering that tissue-based RNA expression detection might be complicated by the non-tumour tissues that are adjacent to tumour cells, we analysed NOS3 mRNA expression in 1457 cell lines derived from 26 tumour types in the CCLE database. Initially, NOS3 expression in different cell lines was checked and the results showed that cell lines from STAD and COAD were the top two cell lines expressing the highest levels of NOS3 mRNA, and cell lines from nerve system tissues (e.g., GBM and neuroblastoma) and bone tissues (e.g., chondrosarcoma and osteosarcoma) expressed relatively lower NOS3 mRNA (Figure 5). Interestingly, NOS3 in STAD was expressed at the highest level both in stomach tissues from TCGA and in stomach cell lines from CCLE. Further analysis of the association between NOS3 mRNA and promoter DNA methylation level showed a weak correlation (Spearman correlation coefficient $=0.1282, p=0.0002$, Figure 5B). Spearman correlation analysis between NOS3 mRNA and copy number did not show statistical significance ( $p=0.1193$, Figure $5 \mathrm{C})$. These results indicated that promoter DNA methylation and copy number variants of the NOS3 gene might not be the main determinant of NOS3 mRNA levels. 


\section{Association between NOS3 expression and drug sensitivity}

To investigate the correlation between NOS3 mRNA expression and drug sensitivity, NOS3 expression in 664 cell lines and drug response to 482 drugs were analysed. Spearman correlation analysis revealed that, only the response to one drug, 'SR8278', was considered to be moderately correlated with NOS3 mRNA expression, with a correlation coefficient greater than 0.3 (Figure 6A). A negative correlation indicated that a better response (smaller response AUC value) was correlated with increased expression of NOS3. 'SR8278' is an antagonist of the transcription factor REV-ERBa, affecting its circadian and metabolic functions. Two other drugs, 'GSK.J4' and 'CIL55A' had a correlation coefficient greater than 0.2 and were also negatively correlated.

Subsequently, Spearman analysis were performed to investigate the correlation of NOS3 expression with drug response separately in cell line types with at least 15 cell lines (a total of 10 cancer cell line types) and the drug response (correlation coefficient >0.3) ratios were calculated. In these cancer cell line types, NOS3 mRNA expression was related to at least one drug, however, the response ratios were quite different and varied depending on the different cell line types (Figure 6B). The response ratios in breast and ovary tumour cell lines were more than $10 \%$. Both breast and ovarian tumour occurred in females and shared some common pathological mechanisms, such as those closely related to female hormones. Therefore, we further analysed the common drugs whose responses were correlated with NOS3 expression in both breast and ovarian tumour cell lines (Figure 6C). The results showed that they shared six common drugs. Among these drugs, LY-2157299, a TGF- $\beta 1$ inhibitor, was strongly associated with NOS3 expression in breast and ovarian tumour cell lines (the Spearman correlation coefficients were -0.64848 and -0.9 , respectively). However, 'SR8278' was not contained.

\section{Discussion}

NOS3 has been found to inhibit apoptosis and promote angiogenesis, proliferation, invasiveness, and immunosuppression of malignant tumours. However, because of the limited number of studies on NOS3 expression in malignant tumours, NOS3 functions in tumour pathogenesis and development are still not fully understood. And the expression pattern of NOS3 and its diagnostic and prognostic potential has not been investigated in a pan-cancer perspective. In this study, the expression level of NOS3 (mainly mRNA) in 30 different normal human tissues, 33 different tumours types as well as their corresponding normal tissues, and 1457 cancer cell lines was systematically analysed, to determine the expression level of NOS3 in tumour and normal tissues and its role in malignant tumours. We also explored its potential association with clinical characteristics (tumour stage, overall survival and drug response).

By analysing NOS3 mRNA levels in normal tissues from GTEx, we found that NOS3 was expressed at the highest level in the spleen and was expressed at the lowest level in the blood. According to the Human Protein Atlas (www.proteinatlas.org) database, the NOS3 protein level in the spleen was also the highest, consisted with our results. And research has reported that NOS3 is mainly upregulated in endothelial progenitor cells (EPCs) of the spleen, exerting beneficial functions on atherosclerosis, angiogenesis, and 
vascular repair $(28,29)$. Analyses of TCGA data showed that NOS3 expression increased in seven tumour tissues compared with their corresponding normal tissues, while its expression decreased in eight tumour tissues. Further analyses of the relationship between NOS3 expression and clinical characteristics also revealed that NOS3 played opposite role depending on different tumour types. NOS3 was reported to promote the angiogenic phenotype and predict poor prognosis in STAD (30). In our research on STAD, NOS3 was differentially expressed in tumour and normal tissues, and expression of NOS3 was the highest among the 33 tumour types and cancer cell lines. Analyses of clinical characteristics also showed that NOS3 predicted poor prognosis, consistent with previous research. These results further confirmed the important role of NOS3 in the development of STAD. In COAD, NOS3 expressed relatively high level in tumour tissues and cancer cell lines, and patients with higher NOS3 levels were diagnosed with a later tumour stage. This was consistent with the observation that L-NIO (a NOS3 inhibitor) inhibited cell growth and angiogenesis in colorectal cancer, and the anti-tumour effect of E7080 (lenvatinib) could be increased after combination with L-NIO in colorectal cancer $(31,32)$. Although the analyses of NOS3 expression from the current TCGA data was not statistically relevant to the prognosis in COAD patients, Marisi et al. found that the NOS3 expression level affected the progression-free survival and overall survival (OS) of metastatic colorectal cancer patients (9). In PRAD, NOS3 expression was higher in tumour tissues, but did not significantly affect overall survival (stage data could not be accessed). In studies reported by Nanni et al., NOS3 overexpression in PRAD promoted the aggressive phenotype of tumour and was related to poor prognosis in patients $(17,18)$. The inconsistent results may be caused by different population and the number of samples. Other tumour types, such as CESC, HNSC, $\mathrm{KICH}, \mathrm{LIHC}, \mathrm{PAAD}, \mathrm{READ}$, and UCEC, also expressed different levels of NOS3 in tumour and normal tissues but did not show a statistically significant influence on clinical characteristics. Therefore, NOS3 expression might have no influence on clinical characteristics. However, some of the possibilities were the limitation of patient number in TCGA or population bias so that the results did not show expected clinical significance. Further verification in larger and more representative populations is needed. Interestingly, both KIRC and KIRP were originally from the kidney, but their pathological mechanism was obviously different (33), and NOS3 showed diametrically opposite expression level and clinical significance in the two tumour types. In addition, there were some tumour types such as ESCA, LUAD, SKCM and MESO that did not differentially express NOS3 in tumour and normal tissues but displayed clinical significance. Previous studies have reported that high NOS3 expression levels promote angiogenesis and cancer cell adhesion to intact microvessels. Microangiogenesis in tumour tissues is widely accepted as an important factor for tumour invasion and distant metastasis. Therefore, NOS3 might be a downstream factor in tumour invasion and metastasis regulation rather than a cancer initiation factor $(7,8)$.

Further analyses in cancer cell lines showed that NOS3 was expressed at quite high levels in COAD and STAD cell lines. Considering that promoter DNA methylation and copy number variation might modify mRNA expression levels, the correlation between NOS3 mRNA levels and the two factors was analysed. However, neither of the two factors showed a strong statistical correlation with NOS3 mRNA, indicating that promoter DNA methylation and copy number variations of the NOS3 gene might not be the main determinant of NOS3 mRNA levels. 
Currently, research on NOS3-targeted medicine is mainly concentrating on cardiovascular and cerebrovascular disease. Many inhibitors and agonists have been found to have satisfactory therapeutic effects. For example, ursolic acid, which has an anti-tumour effect, has been proven to promote NOS3 phosphorylation and inhibit NOS3 uncoupling, thereby preventing doxorubicin-induced cardiac toxicity (34). However, the research on and application of NOS3-targeted medicine in malignant tumours are still extremely limited. The NOS3 inhibitor L-NIO was reported to inhibit COAD cell growth and angiogenesis. Another NOS3 inhibitor, N(G)-nitro-L-arginine methyl ester (L-NAME) was also reported to inhibit PAAD tumour growth (15). In addition, L-NIO could promote the anti-tumour effect of lenvatinib $(31,32)$. The NOS3 level was significantly correlated with outcomes of bevacizumab-based chemotherapy in COAD (9, 35). Unfortunately, bevacizumab, L-NIO, and L-NAME were not included in the CTRP database. Our research showed that 'SR8278', an antagonist of Rev-ErbAa was negatively correlated with NOS3 expression, indicating that NOS3 was the potential target of 'SR8278'. 'SR8278' targeted NR1D1, a nuclear hormone receptor (36), reiterating the potential relationship between NOS3 and NR1D1. Further analyses in different cell line types showed different responses among different tumour types. Drug response ratios were more than $10 \%$ in breast and ovary tumour cell lines. Considering that both breast and ovarian tumour are malignant tumours affected by hormones, and previous research revealed that NOS3 interacts with ER and AR (16-18), common drugs were analysed. LY-2157299, a TGF- $\beta 1$ inhibitor, was strongly associated with NOS3 expression in both breast and ovarian tumour cell lines, indicating that NOS3 expression level influences response of TGF- $\beta 1$ to LY-2157299, and LY-2157299 might inhibit NOS3 expression in a TGF- $\beta 1$ dependent way. Our pan-cancer research suggests that we can further investigate the effect of these target medicines on tumour therapy, especially in patients with cardiovascular and cerebrovascular disease, to maximize the therapeutic effect of drugs.

There are also some limitations in our research. Usually, protein is the final functioning molecule. Our research mainly focused on the mRNA expression level. However, many factors might modulate the translation process. Thus, the mRNA level could not completely represent the protein level. Therefore, more research and analyses at the protein level are need.

\section{Conclusions}

In conclusion, this research showed that NOS3 was a pro- or anticancer factor depending on tumour type. NOS3 expression was different in different tumour types and related to tumour stage and overall survival of patients. Especially, in STAD and COAD, NOS3 expressed high level in tumour tissues and cells, and was related to aggressive tumor stage and poor prognosis of patients, indicating its potential as a biomarker of these two tumour types. In addition, NOS3 expression was related to some therapeutic drugs, such as 'SR8278' and LY-2157299, which warrant further investigation.

\section{Declarations}

Ethics approval and consent to participate: Not applicable. 
Consent for publication: Not applicable.

Availability of data and materials: The datasets analysed during the current study are available in the GTEx (https://www.gtexportal.org/home/), TCGA (; https://portal.gdc.cancer.gov/), CCLE (CCLE; https://portals.broadinstitute.org/ccle/), and CTRP (https://portals.broadinstitute.org/ctrp.v2.1/).

Competing interests: The authors declare that they have no competing interests.

Funding: This research was funded by National Natural Science Foundation of China, grant number 81270036, 30901736; the Plan to Focus on Research and Development from Science and Technology project of Liaoning Province, grant number 2017225029; Science and Technology Plan Project of Shenyang City, grant number 18-014-4-11; and Fund for Scientific Research of The First Hospital Of China Medical University, grant number FHCMU- FSR.

Authors' contributions: Project administration, YZ and ZJ; software, FL and JPZ; statistical analysis, DZ, YC and ZJ; visualization, CJY and YJ; manuscript writing, DZ and YY.

Acknowledgements: The authors thank the colleges in the First Laboratory of Cancer Institute for data collation and statistical analysis

\section{References}

1. Xu W, Liu LZ, Loizidou M, Ahmed M, Charles IG. The role of nitric oxide in cancer. Cell research. 2002;12(5-6):311-20.

2. Fischmann TO, Hruza A, Niu XD, Fossetta JD, Lunn CA, Dolphin E, et al. Structural characterization of nitric oxide synthase isoforms reveals striking active-site conservation. Nature structural biology. 1999;6(3):233-42.

3. Trane AE, Pavlov D, Sharma A, Saqib U, Lau K, van Petegem F, et al. Deciphering the binding of caveolin-1 to client protein endothelial nitric-oxide synthase (eNOS): scaffolding subdomain identification, interaction modeling, and biological significance. The Journal of biological chemistry. 2014;289(19):13273-83.

4. Pritchard KA, Ackerman AW, Ou J, Curtis M, Smalley DM, Fontana JT, et al. Native low-density lipoprotein induces endothelial nitric oxide synthase dysfunction: role of heat shock protein 90 and caveolin-1. Free radical biology \& medicine. 2002;33(1):52-62.

5. Xia N, Daiber A, Habermeier A, Closs El, Thum T, Spanier G, et al. Resveratrol reverses endothelial nitric-oxide synthase uncoupling in apolipoprotein E knockout mice. The Journal of pharmacology and experimental therapeutics. 2010;335(1):149-54.

6. Ying L, Hofseth LJ. An emerging role for endothelial nitric oxide synthase in chronic inflammation and cancer. Cancer research. 2007;67(4):1407-10.

7. Song Y, Zhao XP, Song K, Shang ZJ. Ephrin-A1 is up-regulated by hypoxia in cancer cells and promotes angiogenesis of HUVECs through a coordinated cross-talk with eNOS. PloS one. 
2013;8(9):e74464.

8. Zhang $L$, Zeng M, Fu BM. Inhibition of endothelial nitric oxide synthase decreases breast cancer cell MDA-MB-231 adhesion to intact microvessels under physiological flows. American journal of physiology Heart and circulatory physiology. 2016;310(11):H1735-47.

9. Marisi G, Scarpi E, Passardi A, Nanni O, Ragazzini A, Valgiusti M, et al. Circulating VEGF and eNOS variations as predictors of outcome in metastatic colorectal cancer patients receiving bevacizumab. Scientific reports. 2017;7(1):1293.

10. Penarando J, Lopez-Sanchez LM, Mena R, Guil-Luna S, Conde F, Hernandez V, et al. A role for endothelial nitric oxide synthase in intestinal stem cell proliferation and mesenchymal colorectal cancer. BMC biology. 2018;16(1):3.

11. Maniyar R, Chakraborty S, Suriano R. Ethanol Enhances Estrogen Mediated Angiogenesis in Breast Cancer. Journal of Cancer. 2018;9(21):3874-85.

12. Sharma S, Guru SK, Manda S, Kumar A, Mintoo MJ, Prasad VD, et al. A marine sponge alkaloid derivative 4-chloro fascaplysin inhibits tumor growth and VEGF mediated angiogenesis by disrupting $\mathrm{PI3K} / \mathrm{Akt} / \mathrm{mTOR}$ signaling cascade. Chemico-biological interactions. 2017;275:47-60.

13. Gajalakshmi P, Priya MK, Pradeep T, Behera J, Muthumani K, Madhuwanti S, et al. Breast cancer drugs dampen vascular functions by interfering with nitric oxide signaling in endothelium. Toxicology and applied pharmacology. 2013;269(2):121-31.

14. Lim KH, Ancrile BB, Kashatus DF, Counter CM. Tumour maintenance is mediated by eNOS. Nature. 2008;452(7187):646-9.

15. Lampson BL, Kendall SD, Ancrile BB, Morrison MM, Shealy MJ, Barrientos KS, et al. Targeting eNOS in pancreatic cancer. Cancer research. 2012;72(17):4472-82.

16. Yu S, Jia L, Zhang Y, Wu D, Xu Z, Ng CF, et al. Increased expression of activated endothelial nitric oxide synthase contributes to antiandrogen resistance in prostate cancer cells by suppressing androgen receptor transactivation. Cancer letters. 2013;328(1):83-94.

17. Nanni S, Benvenuti V, Grasselli A, Priolo C, Aiello A, Mattiussi S, et al. Endothelial NOS, estrogen receptor beta, and HIFs cooperate in the activation of a prognostic transcriptional pattern in aggressive human prostate cancer. The Journal of clinical investigation. 2009;119(5):1093-108.

18. Nanni S, Aiello A, Re A, Guffanti A, Benvenuti V, Colussi C, et al. Estrogen-dependent dynamic profile of eNOS-DNA associations in prostate cancer. PloS one. 2013;8(5):e62522.

19. Trachootham D, Chen G, Zhang W, Lu W, Zhang H, Liu J, et al. Loss of p53 in stromal fibroblasts promotes epithelial cell invasion through redox-mediated ICAM1 signal. Free radical biology \& medicine. 2013;58:1-13.

20. Villegas SN, Gombos R, Garcia-Lopez L, Gutierrez-Perez I, Garcia-Castillo J, Vallejo DM, et al. PI3K/Akt Cooperates with Oncogenic Notch by Inducing Nitric Oxide-Dependent Inflammation. Cell reports. 2018;22(10):2541-9.

21. Li Q, Wei X, Zhou ZW, Wang SN, Jin H, Chen KJ, et al. GADD45alpha sensitizes cervical cancer cells to radiotherapy via increasing cytoplasmic APE1 level. Cell death \& disease. 2018;9(5):524. 
22. Su CW, Chien MH, Lin CW, Chen MK, Chow JM, Chuang CY, et al. Associations of genetic variations of the endothelial nitric oxide synthase gene and environmental carcinogens with oral cancer susceptibility and development. Nitric oxide : biology and chemistry. 2018;79:1-7.

23. Zhu Y, Jiang H, Chen Z, Lu B, Li J, Peng Y, et al. The genetic association between iNOS and eNOS polymorphisms and gastric cancer risk: a meta-analysis. OncoTargets and therapy. 2018;11:2497507.

24. Di Salvatore M, Lo Giudice L, Rossi E, Santonocito C, Nazzicone G, Rodriquenz MG, et al. Association of IL-8 and eNOS polymorphisms with clinical outcomes in bevacizumab-treated breast cancer patients: an exploratory analysis. Clinical \& translational oncology : official publication of the Federation of Spanish Oncology Societies and of the National Cancer Institute of Mexico. 2016;18(1):40-6.

25. Smeda M, Kieronska A, Adamski MG, Proniewski B, Sternak M, Mohaissen T, et al. Nitric oxide deficiency and endothelial-mesenchymal transition of pulmonary endothelium in the progression of 4T1 metastatic breast cancer in mice. Breast cancer research : BCR. 2018;20(1):86.

26. Zheng Y, Dai Y, Liu W, Wang N, Cai Y, Wang S, et al. Astragaloside IV enhances taxol chemosensitivity of breast cancer via caveolin-1-targeting oxidant damage. Journal of cellular physiology. 2019;234(4):4277-90.

27. Vivian J, Rao AA, Nothaft FA, Ketchum C, Armstrong J, Novak A, et al. Toil enables reproducible, open source, big biomedical data analyses. Nature biotechnology. 2017;35(4):314-6.

28. Laufs U, Werner N, Link A, Endres M, Wassmann S, Jurgens K, et al. Physical training increases endothelial progenitor cells, inhibits neointima formation, and enhances angiogenesis. Circulation. 2004;109(2):220-6.

29. Gertz K, Priller J, Kronenberg G, Fink KB, Winter B, Schrock H, et al. Physical activity improves longterm stroke outcome via endothelial nitric oxide synthase-dependent augmentation of neovascularization and cerebral blood flow. Circulation research. 2006;99(10):1132-40.

30. Wang L, Shi GG, Yao JC, Gong W, Wei D, Wu TT, et al. Expression of endothelial nitric oxide synthase correlates with the angiogenic phenotype of and predicts poor prognosis in human gastric cancer. Gastric cancer : official journal of the International Gastric Cancer Association and the Japanese Gastric Cancer Association. 2005;8(1):18-28.

31. Altun A, Temiz TK, Balci E, Polat ZA, Turan M. Effects of tyrosine kinase inhibitor E7080 and eNOS inhibitor L-NIO on colorectal cancer alone and in combination. Chinese journal of cancer research $=$ Chung-kuo yen cheng yen chiu. 2013;25(5):572-84.

32. Gao Y, Zhou S, Xu Y, Sheng S, Qian SY, Huo X. Nitric oxide synthase inhibitors $1400 \mathrm{~W}$ and L-NIO inhibit angiogenesis pathway of colorectal cancer. Nitric oxide : biology and chemistry. 2019;83:33-9.

33. Ciccarese C, lacovelli R, Brunelli M, Massari F, Bimbatti D, Fantinel E, et al. Addressing the best treatment for non-clear cell renal cell carcinoma: A meta-analysis of randomised clinical trials comparing VEGFR-TKis versus mTORi-targeted therapies. European journal of cancer (Oxford, England : 1990). 2017;83:237-46. 
34. Mu H, Liu H, Zhang J, Huang J, Zhu C, Lu Y, et al. Ursolic acid prevents doxorubicin-induced cardiac toxicity in mice through eNOS activation and inhibition of eNOS uncoupling. Journal of cellular and molecular medicine. 2019;23(3):2174-83.

35. Ulivi P, Scarpi E, Passardi A, Marisi G, Calistri D, Zoli W, et al. eNOS polymorphisms as predictors of efficacy of bevacizumab-based chemotherapy in metastatic colorectal cancer: data from a randomized clinical trial. Journal of translational medicine. 2015;13:258.

36. Ferder IC, Fung L, Ohguchi Y, Zhang X, Lassen KG, Capen D, et al. Meiotic gatekeeper STRA8 suppresses autophagy by repressing Nr1d1 expression during spermatogenesis in mice. PLoS genetics. 2019;15(5):e1008084.

\section{Figures}

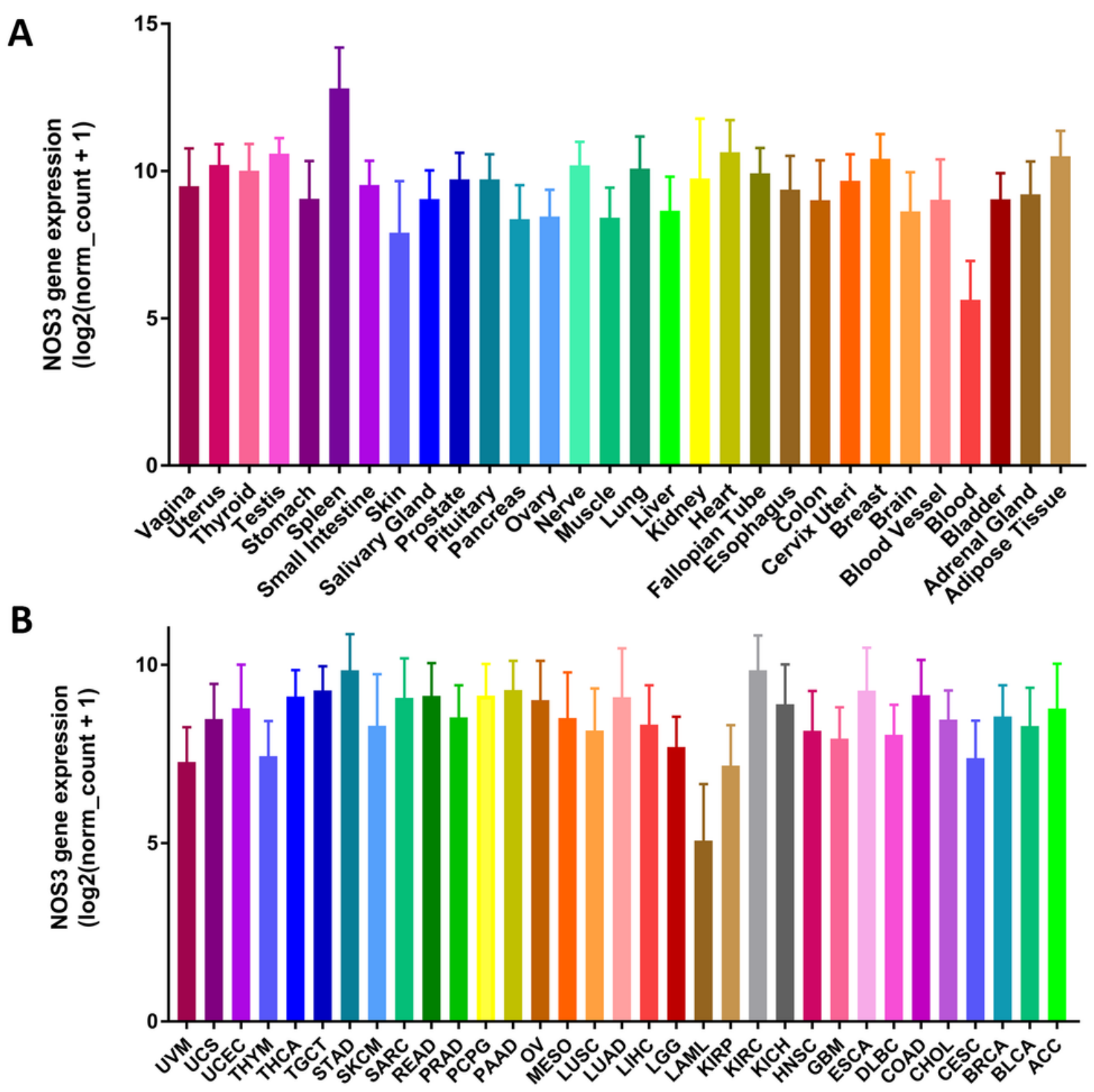

Figure 1 
NOS3 mRNA expression in various normal tissues and tumors. (A) NOS3 expression level in normal tissues from GTEX. (B) NOS3 expression level in tumor tissues from TCGA. The type of NOS3 expression data is RNA-seq RSEM, shown by log2(normalized count +1 ). Each bar represents NOS3 expression among different individuals for a particular tissue type. Error bars represent the mean \pm SD.

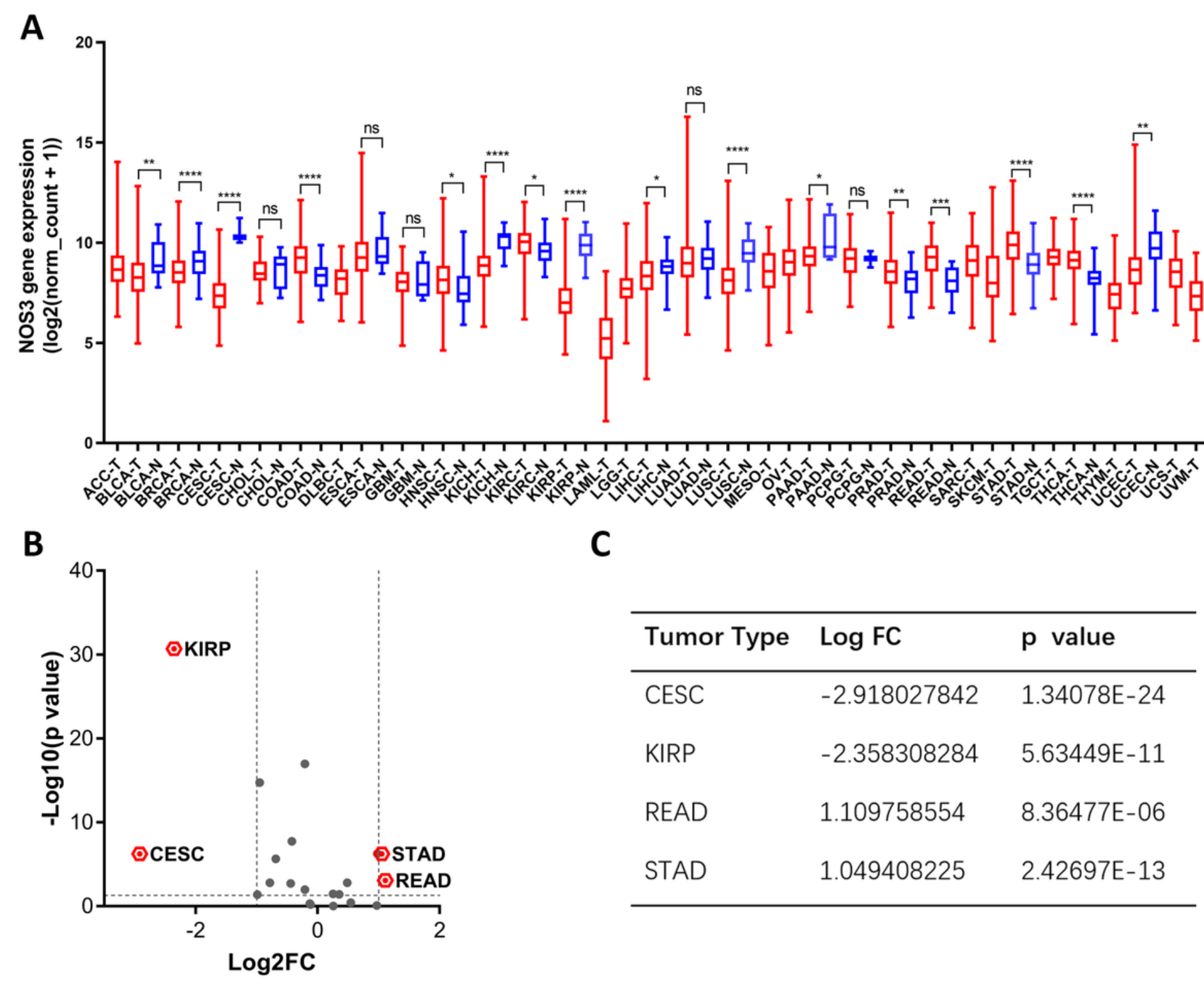

Figure 2

NOS3 is differentially expressed in various tumors and their corresponding normal tissues. (A) NOS3 is differentially expressed between tumor and normal tissues in some cancers from TCGA. Each boxplot represents NOS3 expression (RNA-seq RSEM, log2(normalized count +1 )) across different cancers. Red is for tumors and blue is for normal tissues. The bar represents median expression of tumors or normal tissues and lower and upper box ends represent the 25th and 75th percentile expression. ns, without statistical significance, ${ }^{*} p<0.05,{ }^{* \star} p<0.01,{ }^{* \star *} p<0.001,{ }^{* \star \star \star} p<0.0001$, based on Student's t-test. (B) Scatter plot of log2 FC and minus log10(p-value) across different cancers. The horizontal line on the $Y$ axis represents a $p$ value of 0.05 . Points above the horizontal line have statistical significance. The 
vertical line on the X-axis represents log2 FC was -1 or 1 , respectively. (C) The log $2 \mathrm{FC}$ and $\mathrm{p}$ value in several tumor types, which simultaneously meet the condition that absolute value of log2 FC is larger than 1 and the difference is statistically significant.
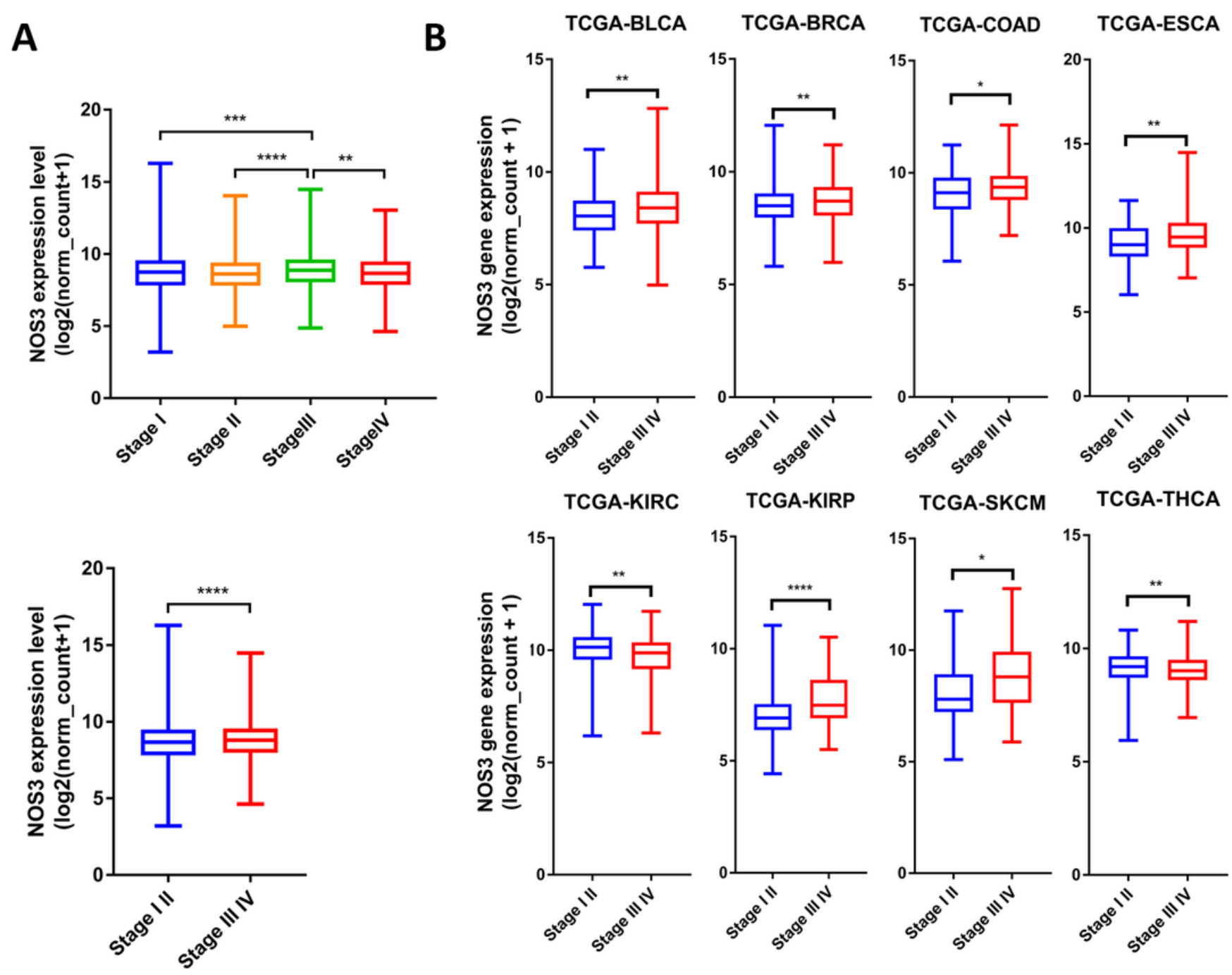

\section{Figure 3}

Association between NOS3 mRNA expression and tumor stage. (A) NOS3 expression (RNA-seq RSEM, log2(normalized count +1)) among stages of all tumor types. (B) NOS3 expression between the early and advanced stages in each separate tumor type. ANOVA test was applied to analyzes across four stages, and a t-test was applied to analyzes in the early stage and advanced stage. ${ }^{*} p<0.05,{ }^{\star \star} p<0.01,{ }^{* \star} p<$ $0.001, \star \star \star \star x p 0.0001$. 
A
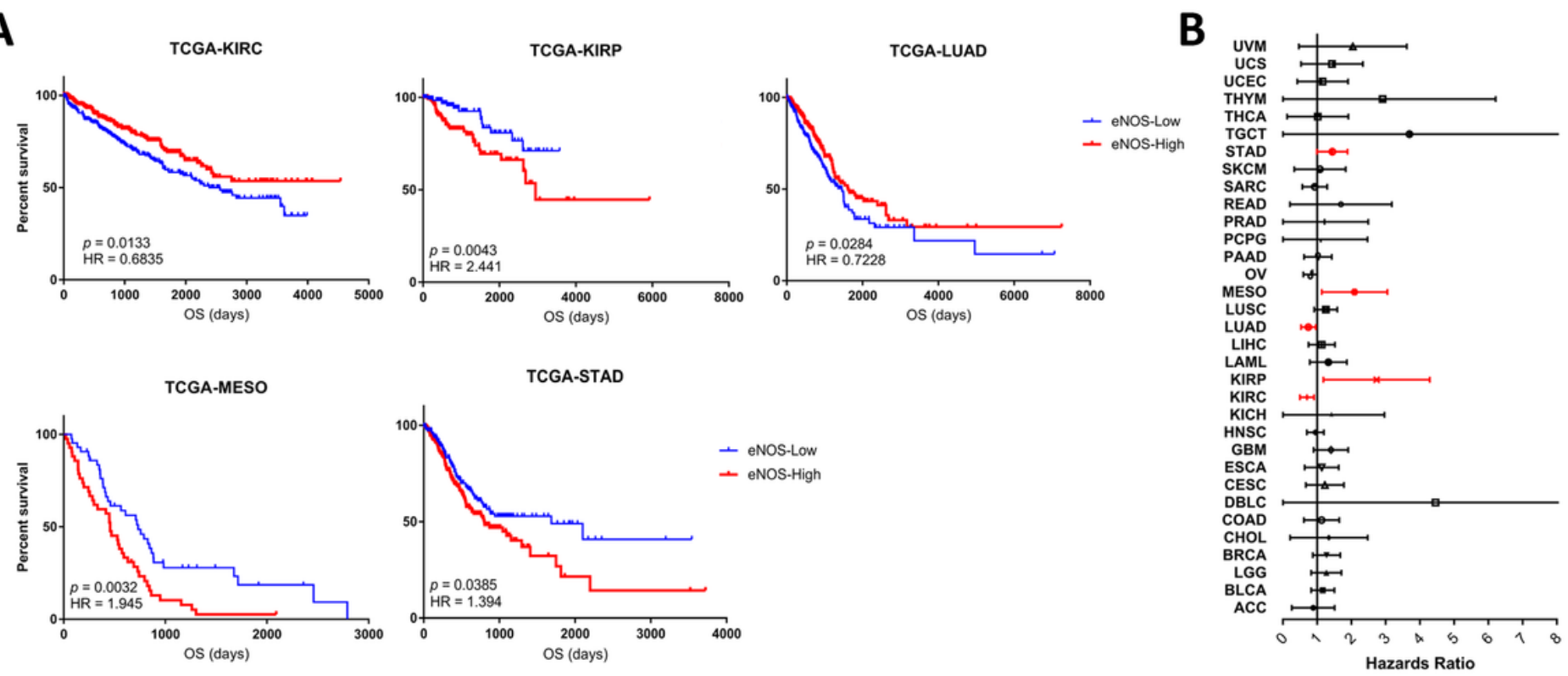

\section{Figure 4}

Association between NOS3 mRNA expression and overall survival. The NOS3 expression level is related to overall survival in several tumor types. In the survival curves, the red line represents high NOS3 expression levels and the blue line represents low NOS3 expression levels. (B) A forest plot for survival association of each cancer is shown. The X-axis is the HR, the small points are the estimate of HR for each tumor and the bar represents the $95 \%$ confidence interval. Cox proportional hazards models were used to evaluate the association of NOS3 expression levels on overall survival. 


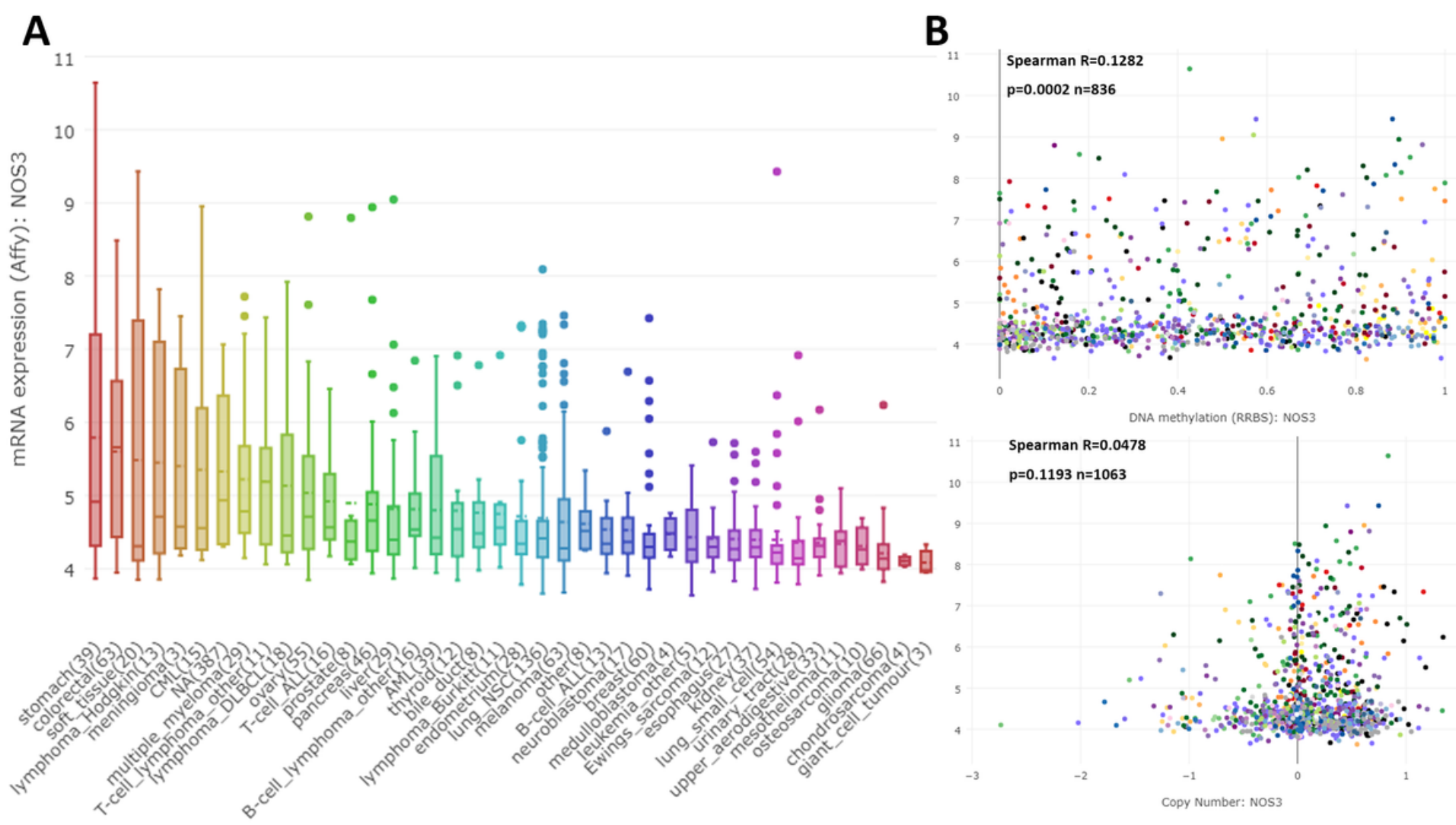

Figure 5

NOS3 mRNA expression in tumor cell lines. (A) NOS3 mRNA expression across different cell lines from CCLE. (B) A scatter plot of promoter DNA methylation and mRNA levels of NOS3 across different cell lines is shown. (C) A scatter plot of copy number variation and mRNA level of NOS3 across different cell lines. The correlation between two variables is analyzed by Spearman analysis. 


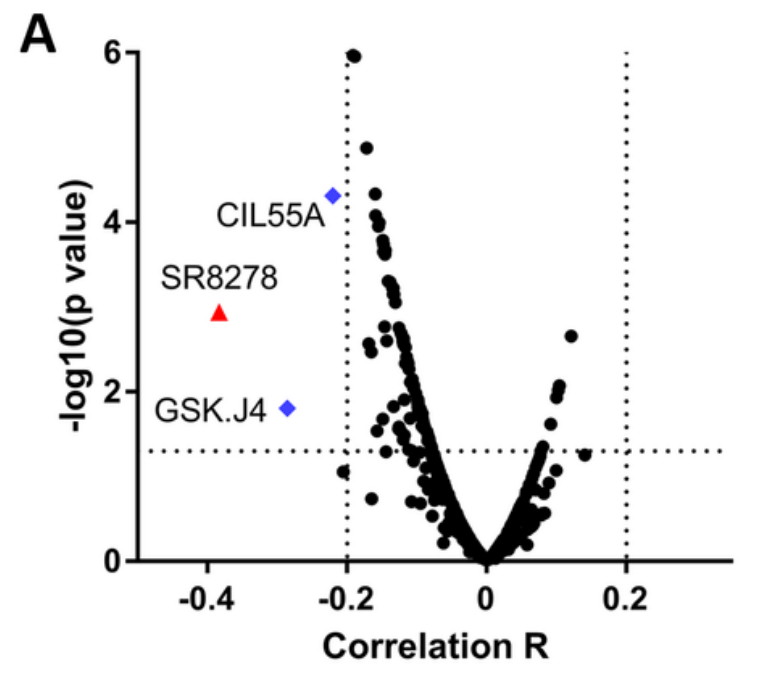

B
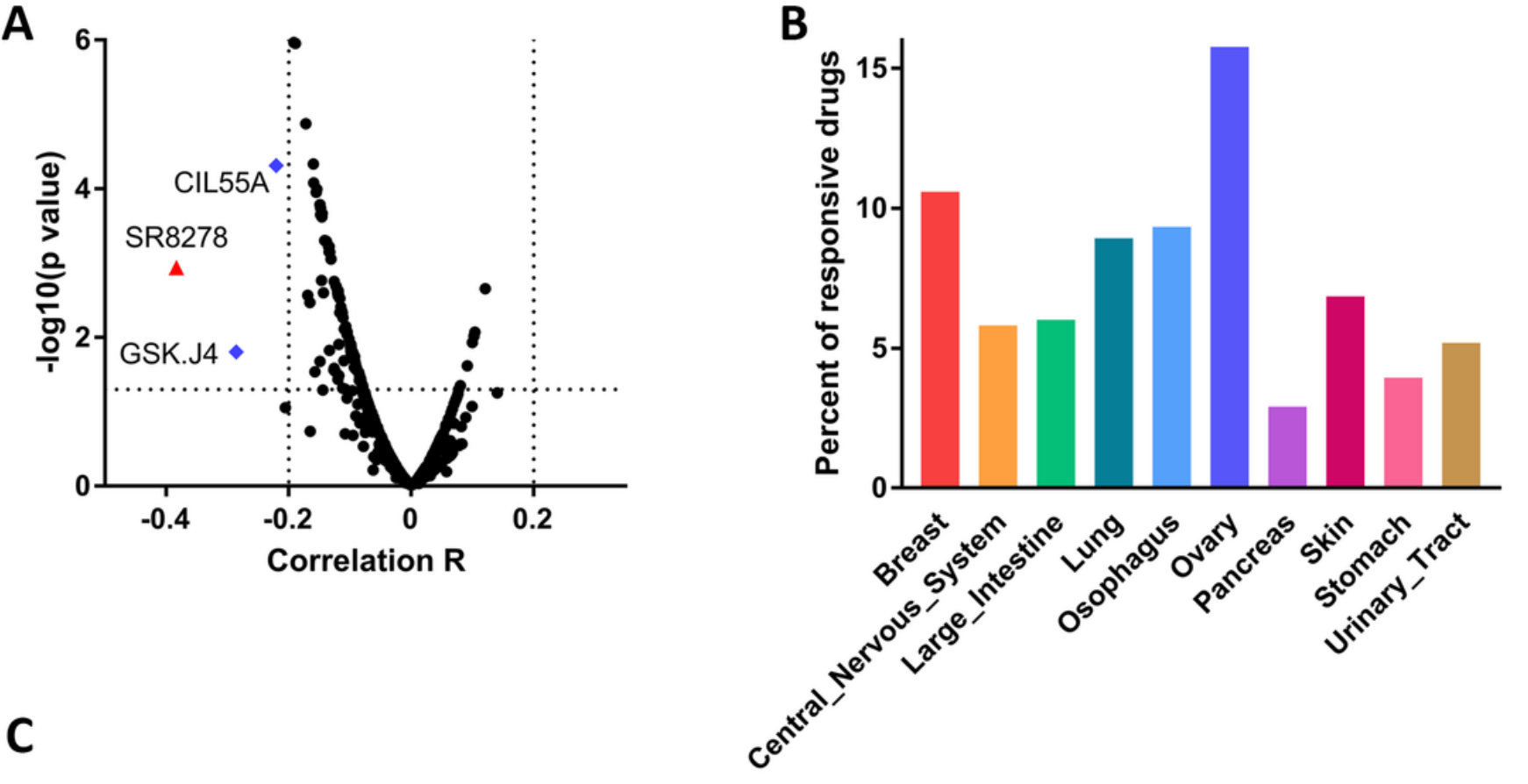

\begin{tabular}{llrc}
\hline \multirow{2}{*}{ Drug name } & Targets & \multicolumn{2}{c}{ Spearman R } \\
\cline { 3 - 4 } & & Breast & Ovary \\
\hline LY-2157299 & Inhibitor of TGF- $\beta 1$ receptor & -0.64848 & -0.9 \\
CIL70 & Unknown & -0.59406 & -0.64239 \\
PF-573228 & Inhibitor of focal adhesion kinase & -0.47186 & -0.45135 \\
Compound 23 citrate & Analog of natural product cortistatin & -0.4568 & -0.58746 \\
Nintedanib & Inhibitor of C-KIT, VEGFRs, PDGFRs, and FGFRs & -0.36926 & -0.38212 \\
N9-isopropylolomoucine & Inhibitor of CDK1/cyclin B and CDK5/p35 complexes & -0.34453 & -0.40456 \\
\hline
\end{tabular}

Figure 6

Association between NOS3 expression and drug sensitivity. (A) Volcano plot of the correlation coefficient and minus log10(p-value) between NOS3 expression in all cell lines and 482 drugs. The majority of correlations are not significant and in negative direction. The correlation coefficient of 'SR8278' is greater than 0.3 (negative, higher expression is correlated with better response represented by smaller AUC). Blue dots are the drugs with correlation coefficients greater than 0.2. (B) The ratio of drugs with correlation coefficients greater than 0.3 and $p$ values less than 0.05 in 10 different cell line types. Cell lines from breast and ovary tumors are responsive to a higher proportion of drugs related to NOS3 expression. (C) Common drugs with correlation coefficients greater than 0.3 and $p$ values less than 0.05 in breast and ovary tumor cells. There are 6 common drugs that respond in relation to NOS3 expression. The targets and correlation coefficient are shown in C.

\section{Supplementary Files}


This is a list of supplementary files associated with this preprint. Click to download.

- supplementarytable.docx 